\title{
Analysis and Discussion on the Effect of Self-Designed Traditional Chinese Medicine and Ice in the Treatment of Sports Injury
}

\author{
Weixing Wang ${ }^{1}$, Guomei Yin ${ }^{2,}$ a \\ ${ }^{1}$ School of Physical Education, Baoshan University, Baoshan, Yunnan 678000, China \\ ${ }^{2}$ Department of Orthopaedics, Baoshan Traditional Chinese Medicine Hospital, Baoshan, Yunnan \\ 678000, China. \\ *,ayinGuomei@163.com
}

Keywords: sports injury; traditional Chinese medicine; ice cold compress.

\begin{abstract}
Objective: To analyze and discuss the effect of self-designed traditional Chinese medicine and ice in the treatment of sports injury. Methods: The study duration was from January 2015 to December 2017 and the 100 participants were selected from patients with acute sports injuries in our outpatient department. These 100 participants were randomly divided into 2 groups with a digital random table method, 50 patients each group. The control group applied safflower oil to the injured part, while observation group added external application of self-designed traditional Chinese medicine combined with ice cold compress on the basis of the control group. The clinical efficacy, pain score, swelling score and life quality score of the two groups were compared. Results: In terms of clinical efficacy, the total effective rate was $96 \%$ in the observation group and $82 \%$ in the control group; the observation group was higher than the control group $(\mathrm{P}<0.05)$. In terms of the pain score and swelling score, the scores of both groups were lower than those before treatment $(\mathrm{P}<0.05)$, while the scores of the observation group were lower than the control group $(\mathrm{P}<0.05)$. In terms of life quality, scores in all aspects of the two groups were higher than that before treatment $(\mathrm{P}<0.05)$, while scores in all aspects of the observation group were higher than that in the control group $(\mathrm{P}<0.05)$. Conclusion: In the treatment of sports injuries, the use of self-designed traditional Chinese medicine externally combined with ice compresses can achieve significant therapeutic effects. This method can effectively relieve pain, swelling and other discomfort, helping to improve the life quality of patients.
\end{abstract}

\section{Introduction}

The sports injury mainly refers to accidental injuries occurring when a person is performing sports, including injuries in skin and bones. Patients are often accompanied by pain and swelling in the injured parts, making their activities restricted partly and their daily life unfavorable, and therefore it is necessary to implement active treatment [1-3]. Ice cold compress and external application of traditional Chinese medicine is the main method for clinical treatment of soft tissue injuries and has been gradually used in the treatment of acute sports injuries in recent years. The purpose of this study was to investigate the role of self-designed traditional Chinese medicine and ice in the treatment of sports injuries. To this end, 100 cases of acute sports injuries were studied.

\section{Data and Methods}

\subsection{General Data.}

The study duration was from January 2015 to December 2017 and the 100 participants were selected from patients with acute sports injuries in our outpatient department. These 100 participants were randomly divided into 2 groups with a digital random table method, 50 cases each group. The age of the control group aged between 25 and 57 and the average age was $(40.59 \pm 12.73)$ years old, including 34 males and 16 females. 23 cases were with injury in the ankle joint, 9 in the lumbar, 8 in the wrist joint, 5 in the knee joint, 3 in the metacarpophalangeal joint, 1 in the elbow joint and 1 in the shoulder. The age of the observation group aged between 23 and 57 and the average age was 
(39.83 \pm 12.54$)$ years old, including 36 males and 14 females. 24 cases were with injury in the ankle joint, 10 in the lumbar, 8 in the wrist joint, 4 in the knee joint, 2 in the metacarpophalangeal joint, 1 in the elbow joint and 1 in the shoulder. Comparing the general data of the two groups, $\mathrm{P}>0.05$, which confirmed this study was comparable.

Both groups of subjects met the inclusion criteria and exclusion criteria in this study. Its inclusion criteria were: (1) Patients were diagnosed with sports injury; (2) They knew the study and were willing to cooperate. Those accompanied by other bone and joint injuries and by internal medicine diseases as well as those who did not cooperate on the study were excluded. The study was approved by the Ethics Committee.

\subsection{Methods.}

The control group adopted safflower oil (Manufacturer: Shanghai Zhonghua Pharmaceutical Nantong Co., Ltd.; SDA License No.: GUOYAOZHUNZI Z32021207). The injured part was coated by moderate safflower oil evenly with a cotton swab, 4 to 6 times a day for 7 days.

The observation group added external application of self-designed traditional Chinese medicine and ice cold compresses on the basis of the control group. The selected traditional Chinese medicinal materials were 100g Safflower, 100g Chinese Angelica, 100g Myrrh, 100g Frankincense, 100g raw Cape Jasmine Fruit, 100g raw Common Floweringquince Fruit, 50g Chinese Thorowax Root, 50g Pseudo-ginseng, 50g Dragon's Blood and 50g raw Rhubarb. The above medicinal materials were ground into a fine powder and added to petrolatum to prepare a paste ointment. The ointment was evenly applied to the gauze which was then covered on the injured part. The dressing was changed once per day for 7 days. When applying cold compress of ice, the right-sized pieces of ice covered with gauze were placed on the injured part with bandage for pressure dressing; the bandage was removed and ice was taken out after 20 minutes of cold compressing, and then the injured part was dressed with a dry clean elastic bandage and sponge, once a day for 7 days.

\subsection{Observation Targets.}

The two groups were compared in the clinical efficacy, pain score, swelling score and life quality score.

Pain score: The Numerical Rating Scale (NRS) was adopted for this item, with a total score of 10 points. The score of 0 meant no pain in the injured part while 10 indicated the most severe pain; the more severe the pain in the injured part, the higher the score [4].

Swelling score: The Visual Analogue Scale (VAS) was adopted, with a total score of 10 points. The score of 0 indicated that the injured part is not swollen while 10 implies "most swollen"; the more serious the swelling of the injured part, the higher the score [5].

Life quality score: The Generic Quality of Life Inventory-74 (GQOL-74) was adopted for this item, covering four aspects, namely physical health, mental health, social function and material life, a maximum score of 100 points for each aspect; the better the quality of life, the higher the score [6].

\subsection{Efficacy Evaluation.}

The evaluation criteria for efficacy were [7]: (1) Healed: the swelling and pain of the injured part basically disappeared and the patient's activities were not limited; (2) Improved: swelling and pain in the injured part was reduced, but the patient still felt pain when the injured part was pressed or during exercise, and activities were also limited to some extent; (3) Ineffective: the swelling and pain was not relieved or even worsened, and activities are severely limited. Total Efficiency $=($ Healing + Improved $)$ Number of cases / Total number of cases $\times 100 \%$.

\subsection{Statistical Analysis.}

SPSS19.0 was employed and $\chi^{2}$ test was applied on enumeration data, presented as cases, $\%$. The between-group and in-group measurement data were respectively analyzed by two independent sample t-test and paired t-test, which were expressed as mean \pm standard deviation; it had statistically significant when $\mathrm{P}<0.05$. 


\section{Results}

\subsection{The Clinical Efficacy Comparison of the Two Groups.}

In terms of clinical efficacy, the total effective rate was $96 \%$ in the observation group and $82 \%$ in the control group. It can be seen from the comparison between the two groups that the observation group was higher than the control group $(\mathrm{P}<0.05)$, as shown in Table 1:

Table 1 the clinical efficacy comparison of the two groups [cases (\%)]

\begin{tabular}{cccccc}
\hline Group & cases $(\mathrm{n})$ & Healed & Improved & Ineffective & Total efficiency \\
\hline Control group & 50 & $19(38 \%)$ & $22(44 \%)$ & $9(18 \%)$ & $41(82 \%)$ \\
Observation group & 50 & $24(48 \%)$ & $24(48 \%)$ & $2(4 \%)$ & $48(96 \%)^{*}$ \\
\hline
\end{tabular}

Note: * indicates comparing with the control group, $\mathrm{P}<0.05$

\subsection{Comparison of the Pain Score and Swelling Score between the Two Groups.}

In terms of pain scores and swelling scores, the scores of both groups were lower than before the treatment $(\mathrm{P}<0.05)$; the scores of the observation group were lower than the control group $(\mathrm{P}<0.05)$, as shown in Table 2.

Table 2. Comparison of the pain score and swelling score between the two groups $(\bar{x} \pm s$, points)

\begin{tabular}{cccc}
\hline Group & Time & Pain score: & Swelling score: \\
\hline \multirow{2}{*}{ Control group $(\mathrm{n}=50)$} & Before treatment & $5.62 \pm 1.57$ & $5.75 \pm 1.54$ \\
& After treatment & $4.08 \pm 1.23 \#$ & $4.22 \pm 1.31 \#$ \\
Observation group $(\mathrm{n}=50)$ & Before treatment & $5.41 \pm 1.62$ & $5.53 \pm 1.59$ \\
& After treatment & $2.86 \pm 1.07 \# *$ & $2.90 \pm 1.12 \# *$ \\
\hline
\end{tabular}

Note: \#indicates comparing with that before treatment; ${ }^{*}$ indicates comparing with the control group, $\mathrm{P}<0.05$.

\subsection{Comparison of Life Quality Scores between the Two Groups.}

In terms of life quality, scores of the two groups in all aspects were higher than that before treatment $(\mathrm{P}<0.05)$; scores of the observation group in all aspects were higher than that in the control group $(\mathrm{P}<0.05)$, as shown in Table 3.

Table 3 Comparison of life quality scores between the two groups ( $\bar{x} \pm s$, point)

\begin{tabular}{cccccc}
\hline Group & Time & Physical health & Physical health & Material life & Social function \\
\hline $\begin{array}{c}\text { Control group } \\
(\mathrm{n}=50)\end{array}$ & $\begin{array}{c}\text { Before } \\
\text { treatment } \\
\text { After } \\
\text { treatment } \\
\text { Before }\end{array}$ & $74.53 \pm 7.42$ & $70.35 \pm 6.90$ & $73.08 \pm 8.21$ & $69.59 \pm 7.19$ \\
& $80.58 \pm 7.93 \#$ & $76.72 \pm 7.82 \#$ & $79.28 \pm 8.57 \#$ & $75.92 \pm 7.85 \#$ \\
$\begin{array}{c}\text { Observation } \\
\text { group (n=50) }\end{array}$ & $\begin{array}{c}\text { treatment } \\
\text { After } \\
\text { treatment }\end{array}$ & $89.69 \pm 7.85$ & $70.56 \pm 6.74$ & $73.37 \pm 8.04$ & $69.87 \pm 7.64$ \\
\hline
\end{tabular}

Note: \#indicates comparing with that before treatment; * indicates comparing with the control group, $\mathrm{P}<0.05$.

\section{Discussion}

Sports injury mainly refers to an accidental injury that occurs when people exercise in sports and has a high incidence. The patient generally suffers pain and swelling then. Slight injuries mainly affect the skin and muscles, while severe injuries may injure the patient's bones and joints. These injuries may lead to limited physical activity of the patient, interfere with the patient's daily life, work 
and study and make them feel pain physically and psychologically. If the patient fails to treat, it may also develop from the acute sports injury to the chronic sports injury which is prone to recurrent attacks, seriously affecting the life quality of the patient. Therefore, it is necessary to actively treat sports injuries clinically.

In the past, safflower oil was applied in the clinical treatment of sports injuries. This could relieve the swelling and pain in the injured part to a certain extent, but its curative effect is not ideal. In recent years, cold compress with ice has been gradually used in the treatment of sports injuries. The ice cold compress is mainly achieved by placing ice cubes on the injured part. This method, to a certain extent, can promote the temperature decrease of local tissue and vasoconstriction, which is conducive to reducing local congestion, suppressing nerve sensation and play a role in hemostasis, analgesia, etc.; however, the time for ice cold compress should be controlled within 20 minutes to avoid frostbite [8-9].

In traditional Chinese medical science, sports injuries belong to the category of "the injury of tendon and muscle". Chinese medicine treatment of sports injury advocates starting from the overall perspective to give comprehensive consideration of the patient's bones, blood, meridians and organs, and the principle of treatment is clearing heat, cooling blood and promoting blood circulation to remove blood stasis [10]. In this study, the observation group used self-designed traditional Chinese medicine for external application, and the herbs in the prescription had their own effects. Specifically, Safflower, Chinese Angelica and raw Common Floweringquince Fruit can Shujingtongluo and activate blood circulation; Myrrh, Frankincense and Panax Notoginseng is conducive to relieving swelling and pain and promoting blood circulation and tissue regeneration; Bupleurum can reconcile superficies and interior; Dragon's Blood can promote blood circulation and relieve pain; raw Rhubarb is conducive to clearing heat and detumescence; raw Cape Jasmine Fruit can be used for removing heat to cool blood, detoxifying and discharging fire. The combination of these medical materials can play a role in removing heat, promoting blood circulation and relieving swelling and pain. In modern pharmacology, the prescription can also improve microcirculation and inhibit inflammatory exudation, helping to reduce swelling and inflammation.

It is found in this study that the total clinical efficiency in the observation group (96\%) was higher than that in the control group $(82 \%), \mathrm{P}<0.05$. The pain score and swelling score of the observation group after treatment were lower than those of the control group $(\mathrm{P}<0.05)$, and the life quality scores in all aspects were higher than that in the control group $(\mathrm{P}<0.05)$. This fully shows that it is feasible to treat sports injuries with the external application of traditional Chinese medicines combined with ice cold compress, because it can effectively promote pain and swelling relief in patients in the injured part, help to enhance the therapeutic effect and improve the quality of their life.

To sum up, in the treatment of sports injuries, the use of external application of self-designed traditional Chinese medicine and cold compress of ice can effectively relieve pain, swelling and other discomfort, achieving significant treatment effects to improve the life quality of patients.

\section{References}

[1]. Gorlitz T, Stein S, Pfeifer K, et al. Functional ankle instability as a risk factor for osteoarthritis: Using T2-mapping to analyze early cartilage degeneration in the ankle joint of young athletes[J]. Osteoarthritis and cartilage,2014,22(10):1377-1385.

[2]. Prado MP, Mendes AAM, Amodio DT, et al. A comparative, prospective, and randomized study of two conservative treatment protocols for first-episode lateral ankle ligament injuries [J].Foot and ankle international, 2014, 35(3):201-206.

[3]. Yuen Jin, Mo Shenzhen, Yue Yunqing et al. Clinical Observation of Warm Acupuncture Therapy Combined with External Application of Chinese Medicine in the Treatment of Acute Sports-induced Ankle Injury [J]. Journal of Clinical Acupuncture and Moxibustion, 2015, 31(2): 29-32. 
[4]. $\mathrm{Cu}$ Xiao. Experimental Study of Imitating Exercise-induced Soft Tissue Injury after Compression Dressing [D]. Hunan Normal University, 2014.

[5]. Siu Xianlun, Wang Ming, Xie Weifeng, et al. Observation of the Effect of Chinese Medicine Speed Spectrum Medical Band on the Treatment of Sports System Injuries [J]. Practical Journal of Medicine \& Pharmacy, 2013, 30(5): 439-440.

[6]. Huang Honie. Study on the Effect of Traditional Huoxue Shengji Chinese Medicine (Astragalus and Angelica Compound) on the Recovery ofRat Skeletal Muscle Micro-damage [D]. Qufu Normal University, 2015.

[7]. Lei Xiao. Influence of different time points of ice bag compress on swelling and pain in patients with ankle fracture combined with soft tissue injuries [J]. Journal of Traumatic Surgery, 2017, 19(12): 920-923.

[8]. Zhou Li. Clinical Research on Early Ice Compress Combined with Shangkesan in the Treatment for Acute Closed Soft Tissue Injury in Military Training [J]. Shanxi Medical Journal, 2013, 42(17): 1040-1042.

[9]. Zhao Yeanling, Xiao Qing bang, Jiang Zigong et al. Effect of different ice deposition methods on treatment of acute closed soft tissue injury [J]. China Journal of Modern Medicine, 2016, 26(20): $1-5$.

[10]. Yang Tonga. Application Effect of External Application of Chinese Herbs Combined with Needle Warming Moxibustion in Acute Sports Ankle Injury [J]. Modern Medicine \& Health, 2017, 33(20): 3177-3178. 